\title{
Streamers Variability Investigation of a Radio- Frequency Corona Discharge in an Optical Access Engine at Different Speeds and Loads
}

\author{
Federico Ricci ${ }^{1 *}$, Roberto Martinelli ${ }^{1}$, Luca Petrucci $^{1}$, Gabriele Discepoli ${ }^{1}$, Carlo Nazareno \\ Grimaldi ${ }^{1}$ and Stefano Papi $^{2}$ \\ ${ }^{1}$ Università degli Studi di Perugia, Dipartimento di Ingegneria, 06125 Perugia (PG), Italy \\ ${ }^{2}$ Federal-Mogul Powertrain Italy a Tenneco Group Company, Stabilimento Ignition, 41012 Carpi \\ (MO), Italy
}

\begin{abstract}
Engine research community interest in the Radio-Frequency corona-based ignition systems is currently gaining in importance mainly due to their capability to ensure robust combustion at challenging operating conditions such as very lean mixture and/or high EGR dilution. The benefits of Corona low-temperature plasma foster the early flame development thanks to combustion precursors production and to a more energetic and volumetric discharge, resulting in a larger amount of involved mixture. The corona discharge generates ionizing waves, named streamers, whose temporal and spatial variability in orientation, length and branching can affect the combustion onset and, therefore, the engine cycleto-cycle variability.

In this work, the discharge natural luminosity of a RF corona igniter, characterized by four tips electrodes, was recorded in an optically accessible engine via high-speed camera detection. A preliminary statistical analysis of the spatial and temporal streamer variability was performed by operating in motored conditions. Four different engine speeds and two different loads were explored in order to deeply investigate the streamer behaviour at diverse engine operating conditions.

A comparison between a motored and a lean operating condition is also proposed to analyse, at a specific engine speed, the mixture influence on the streamers propagation before the start of the combustion.
\end{abstract}

\section{Introduction}

Currently, Internal Combustion Engines (ICEs) are forced to decrease the amount of pollutant emissions and fuel consumption while maintaining high performance [1,2]. Spark ignition (SI) engines actual trends to obtain a cleaner and more efficient combustion are focusing on innovative strategies such as water injection, turbocharging, high exhaust gas recirculation (EGR) dilution, and/or lean combustion $[3,4,5]$. At these operating conditions, where high released energy is required for mixture ignition, conventional spark plugs have shown its limits in obtaining robust combustion onsets with low cycle-to-cycle variability [6]. To improve ignition capability, spark-based solutions like multiple strikes discharge [7] or high-energy discharge [8] have shown issues due to reduced sparkplug lifetime. Automotive research has currently found in low-temperature plasma (LTP)-based ignition 
systems a valid solution to improve ignition capability especially in lean and/or diluted conditions $[9,10]$. LTP igniters enhance the combustion not only via thermal effect but also via kinetic and transport effects [11]. Among LTP technologies, one of the most promising solution is represented by radio-frequency (RF) corona streamer-type igniter (CSI) $[12,13]$. CSI discharge is based on the production of a strong electric field amplified through needles/ tips to generate filaments, named streamers [14]. These ionization waves propagate from the device-tips to the counter-electrode represented by the engine walls or by piston head, thus resulting in a larger amount of involved mixture [15]. Calorimetry tests also showed CSI capability to release into the medium higher thermal energy with respect to the one released by a conventional spark igniter [16].

Streamers behaviour is quite different depending on pressure level, mixture composition and igniter setting parameters (mainly electrode voltage $[16,17]$ ); these features make the corona discharge a highly random phenomenon [18].

Therefore, temporal and spatial variability of the streamer in orientation, branching and length can affect the combustion onset and, consequently, the engine cycle-to-cycle variability [19].

Within this context, the present work aims at investigating the steamers length evolution during a CSI discharge in an optical access engine at different testing conditions. Evidences link the streamers penetration to the thermal energy released into the medium and to the production of radicals and excited species via ionization and excitation of the mixture [18]. The larger the amount of charge involved the higher the combustion robustness and the lower the cycle-to-cycle variability.

Preliminary analysis of the spatial and temporal streamer variability was performed at motored conditions, by using different engine speeds and loads.

A comparison between motored and lean operating conditions is also provided to analyse, before the combustion onset, the influence of the mixture on the streamers propagation.

\section{Experimental setup}

\subsection{RF corona igniter}

In this work, a Tenneco ACIS (Advanced Corona Ignition System) streamer-type igniter (CSI) was used to carry out the experimental campaign (Fig.1). A dedicated control unit powers the igniter assembly with an alternating RF current at about $1.04 \mathrm{MHz}$, corresponding to the resonance frequency of the equivalent RLC circuit [20].

The main control parameters of the discharge are the driving voltage $V_{d}$ and the activation time $t_{\text {on }}[34]$, which are respectively correlated to the peak electrode voltage $\left(V_{e}\right)$ and to the discharge duration [16].

In the CSI configuration the streamers start from four-tip electrode and propagate into the medium towards the counter-electrode (Fig.1).

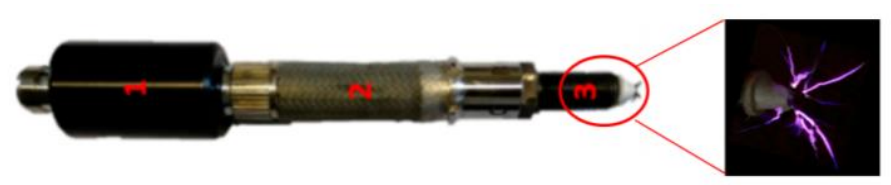

Fig.1. Representation of the streamers development produced by the corona-streamer type igniter ( $1=$ inductor, $2=$ connection, $3=$ firing-end $)$ 


\subsection{Optical Access Engine}

Measurements were carried out on a 500cc single-cylinder engine (Fig.2, (a)) with four valves, pent-roof combustion chamber, and a reverse tumble intake port system which is designed to operate in Direct Injection (DI) or Port Fuel Injection (PFI). The tests were conducted in Port Fuel Injection (PFI) mode with the igniter centrally located. A prolonged piston was used to locate a 45-degree mirror to allow the optical access from the bottom. A $60 \mathrm{~mm}$ diameter quartz was mounted on the piston-head. Conventional rings have been replaced by Teflon graphite rings, as lubrification would hinder high-quality visualization.

A Vision Research Phantom V710 high-speed CMOS camera was used to record the streamers evolution inside the chamber.

A piezoresistive transducer (Kistler 4075A5) on the intake measures the intake port pressure and a piezoelectric transducer (Kistler $6061 \mathrm{~B}$ ) on one side of the chamber measures the in-cylinder pressure. The engine speed control both at motored and firing condition was ensured by an AVL 5700 dynamic brake. The energizing time of the injector and the ignition timing was controlled by a research ECU (Athena GET HPUH4) that sends a trigger signal to the igniter control unit.

In firing mode, the air-fuel ratio $(\lambda)$ was set by reducing or increasing the fuel with fixed throttle position, in order to maintain the same turbulence level inside the combustion chamber. Standard European market gasoline (E5, with $\mathrm{RON}=95$ and $\mathrm{MON}=85)$ was injected at a fixed absolute pressure of 5 bar by means of a port fuel injector (Weber IWP092).

A Kistler Kibox (Fig.2, (b)) combustion analysis system (temporal resolution of 0.1 $\mathrm{CAD}$ ) acquired the pressure signals, the absolute crank angular position (measured by an optical encoder (AVL 365C)), the $\lambda$ (measured by a fast lambda probe at the exhaust (Horiba MEXA- 720, accuracy of $\pm 2.5 \%$ )), the ignition signal (from ECU), and the trigger signal used for synchronization purposes. A total of 63 consecutive events were recorded for each operating point tested.

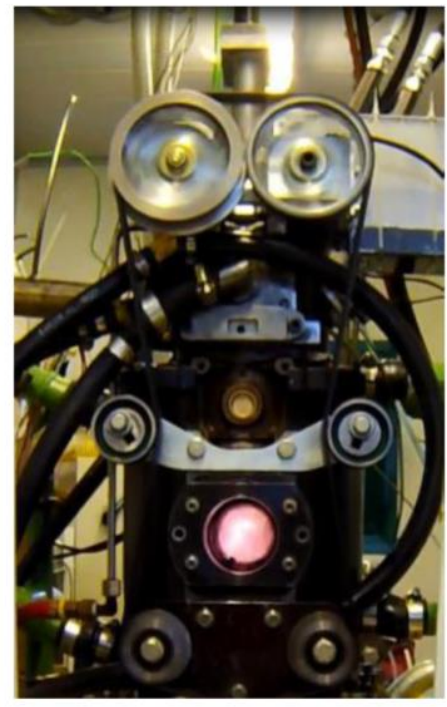

(a)

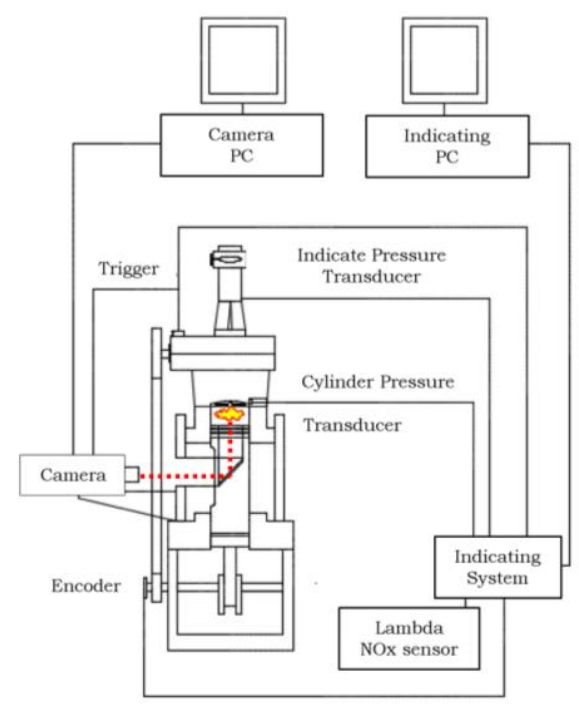

(b)

Fig.2. (a) Optical access engine in firing condition and (b) experimental apparatus to perform the optical access engine tests. 


\subsection{Imaging System}

The Vision Research Phantom V710 high-speed CMOS camera used to record the natural luminosity of the streamers was coupled with a Nikon $55 \mathrm{~mm} \mathrm{f} / 2.8$ lens (Table1). The synchronization between imaging data and indicating ones was ensured by a common trigger signal derived from an automotive camshaft position sensor (Bosch 0232103052). The high-speed camera starts recording when the rising edge of the trigger signal is detected. A tuneable pre-trigger length allowed to set a number of frames to be acquired even before the rising edge. Each frame was composed of $256 \times 256$ pixel to magnify the acquisition around the igniter main electrode. Maximum allowable sampling rate of $79 \mathrm{kHz}$ and minimum exposure time of $12.16 \mu$ s were chosen for the herein analysis. A total of 63 consecutive events were recorded for each operating point tested.

Table1. High-speed camera settings.

\begin{tabular}{|l|l|l|}
\hline Feature & Unit & Value \\
\hline Image Resolution & pixel & $256 \times 256$ \\
\hline Spatial Resolution & $\mu \mathrm{m} /$ pixel & 107 \\
\hline Bit Depth & bit & 8 \\
\hline Exposure Time & $\mu \mathrm{s}$ & 12.16 \\
\hline Sampling Rate & $\mathrm{fps}$ & 79,000 \\
\hline Number of consecutive events recorded & - & 63 \\
\hline
\end{tabular}

\section{Methods}

Image processing was carried out by means of in-house MATLAB scripts, together with the use of MATLAB built-in functions. All the procedures needed to evaluate the streamer luminosity and variability can be found in our previous work [18]. For sake of completeness, here are presented the main steps of the analysis.

\subsection{Preliminary Operations}

- Average Background Computation and Removal: the mean grey level of the 20 frames before the start of discharge were computed and subtracted from each frames of the recorded series (Fig.3 (a)).

- Equalization: grey levels were no longer displayed from 0-255 (maximum grey level with 8-bit resolution), but only up to 20 to highlight both bright frames and dark (Fig.3 (b)).

- Filtering: the noise reduction was performed by using two-dimensional (2D) Gaussian filter with a standard deviation of 1.5 because it was considered a good compromise between boundary conservation and noise reduction (Fig.3 (c)).

- Thresholding and Binarization: for all test cases a fixed threshold (6 out of 255) was chosen to distinguish the level above which a pixel is actually considered as streamers and below which it is background. After that, each image was converted into black (no streamer-area detected) and white (streamer-area detected) (Fig.3 (d)). 
- Quadrant Selection to distinguish the different behaviour of the streamers generated by the four-tip electrode, each frame was divided into four quadrants, for separately analysing them (Fig.3 (e)).
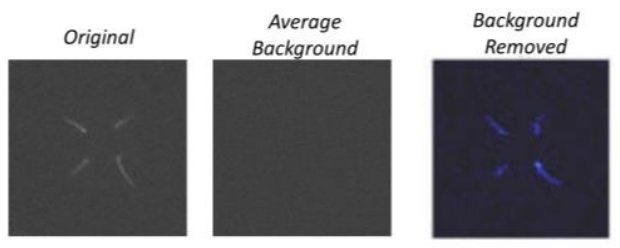

(a)

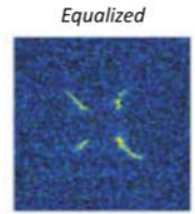

(b)

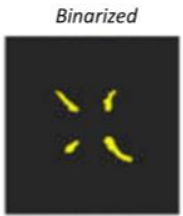

(d)

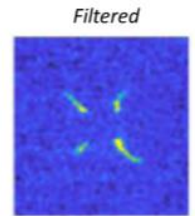

(c)

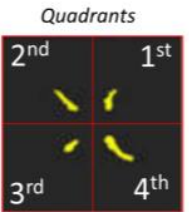

(e)

Fig.3. Preliminary pre-processing procedure for streamers analysis.

\subsection{Streamer Penetration Determination}

After the binarization process, the algorithm determined, for each frame, the streamer penetration $\mathrm{P}$ defined as the maximum distance between the streamers head and the corresponding tip from which it was generated (Fig.4). Fig.4 shows the temporal evolution of the penetration $\mathrm{P}$ ( $4^{\text {th }}$ quadrant), for all the 63 events acquired (grey lines) and the corresponding mean value (red line).

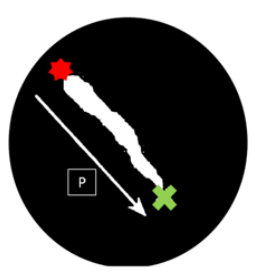

(a)

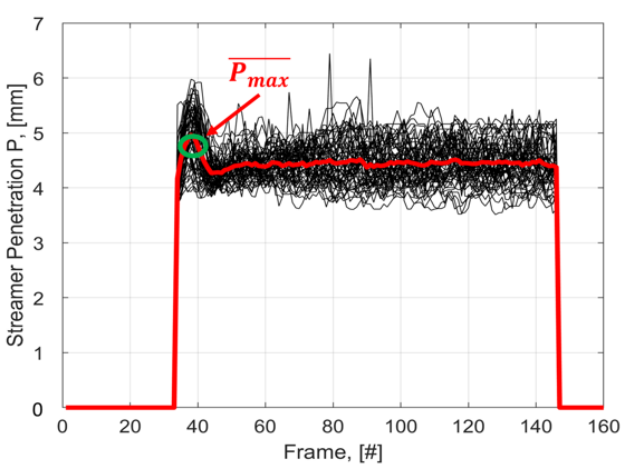

(b)

Fig.4. (a) Streamer penetration $P$ detection. The red asterisk represents the igniter tip position, while the green cross represents the streamer's head point. (b) Temporal evolution of the penetration P for a certain number of streamers (black lines). Circular red marker indicates the max streamer penetration of the mean discharge event (red lines). 
Note that the uncertainty in identifying the streamer penetration for each frame corresponded to \pm 1 pixel, i.e., $0.107 \mathrm{~mm}$ (according to Table1), which is negligible if compared to the maximum penetration values.

\section{Test Campaign}

All the tests were performed with the ignition timing set to obtain, at discharge onset, a fixed in-cylinder pressure of 6 bar.

Tests were carried out at four different speeds and two different loads, with the engine operating in motored conditions (Table2). At $1000 \mathrm{rpm}$, low load (from now on LL) condition was characterized by IMEP=4.5 bar @ $\lambda=1$ while the medium load (from now on $\mathrm{ML})$ one by IMEP $=5.9$ bar $@ \lambda=1[15]$.

Table2. Tests carried out with the engine in motored condition.

\begin{tabular}{|c|c|c|}
\hline \multirow{2}{*}{ Engine Speed, [rpm] } & \multicolumn{2}{|c|}{ Engine Load } \\
\cline { 2 - 3 } & Low & Medium \\
\hline 500 & $\square$ & $\square$ \\
\hline 800 & $\square$ & $\square$ \\
\hline 1000 & $\square$ & $\square$ \\
\hline 1200 & $\square$ & $\square$ \\
\hline
\end{tabular}

Fired condition was also analysed to investigate the mixture influence on the CSI discharge evolution before the start of the combustion.

According to the optical engine operating stable limit, an ultra-lean condition was chosen (i.e. $\lambda=1.6[12]$ ) to offer a comparison with a motored condition, at the same engine speed and load (Table3).

Table3. Comparison between an ultra-lean fired condition and motored one

\begin{tabular}{|c|c|c|c|}
\hline \multirow{2}{*}{ Engine Speed, [rpm] } & \multirow{2}{*}{ Engine Load } & \multicolumn{2}{|c|}{ Condition } \\
\cline { 3 - 4 } & Low & $\square$ & $\square$ \\
\hline 1000 & & $\square$ & $\square$ \\
\hline
\end{tabular}

In each test, maximum allowed corona duration $t_{\text {on }}=1500 \mu \mathrm{s}[12,15]$ was used in order to deeply investigate the corona streamer evolution as pressure increases. For instance, at $1000 \mathrm{rpm}, \mathrm{t}_{\mathrm{on}}=1500 \mu \mathrm{s}$ corresponds to $9 \mathrm{CAD}$.

\section{Results and Discussion}

\subsection{Motored Condition}

The in-cylinder pressure value $\mathrm{P}_{\text {cyl }}$ is a function of both engine speed and load. According to the operating conditions (Table2), it was necessary to determine, case by case, the right IT value in order to maintain the start of discharge as fixed around $p_{\mathrm{ch}}=6 \mathrm{bar}$. The results of preliminary tests are reported in Table4. For each case is shown the corona duration in Crank Angle Degree (CAD), according to the speed engine reference, and the corresponding internal pressure interval under which the igniter operated. 
Table4. Ignition timing for each case analysed and corresponding working pressure interval

\begin{tabular}{|c|c|c|c|c|}
\hline \multirow{2}{*}{ Engine Load } & $\begin{array}{c}\text { Engine Speed, } \\
{[\mathbf{r p m}]}\end{array}$ & $\begin{array}{c}\mathbf{I T}, \\
{[\mathbf{C A D} \text { aTDC] }}\end{array}$ & $\begin{array}{c}\mathbf{t}_{\text {on }}, \\
{[\mathbf{C A D}]}\end{array}$ & $\mathbf{P}_{\text {cyl }}$ (from-to), [bar] \\
\hline \multirow{4}{*}{ Low } & 500 & -43 & 4.5 & $6.00-6.83$ \\
\cline { 2 - 5 } & 800 & -39 & 7.2 & $6.08-7.43$ \\
\cline { 2 - 5 } & 1000 & -38 & 9 & $5.97-7.66$ \\
\cline { 2 - 5 } & 1200 & -36 & 10.5 & $5.99-7.93$ \\
\hline \multirow{4}{*}{ Medium } & 500 & -49 & 4.5 & $5.96-6.68$ \\
\cline { 2 - 5 } & 800 & -47 & 7.2 & $6.01-7.81$ \\
\cline { 2 - 5 } & 1000 & -47 & 9 & $6.10-8.28$ \\
\cline { 2 - 5 } & 1200 & -47 & 10.5 & $6.00-8.65$ \\
\hline
\end{tabular}

The higher the engine speed the wider the in-cylinder pressure range under which the igniter is forced to work. Moreover, at the same $\mathrm{P}_{\text {cyl }} @$ IT, the greater the load the larger the growth rate of the $\mathrm{P}_{\mathrm{cyl}}$ curve (an example is reported in Fig.5). Consequently, the igniter has to deal with higher pressure levels at ML with respect to the LL because of the larger amount of air trapped inside the cylinder during the engine intake phase.

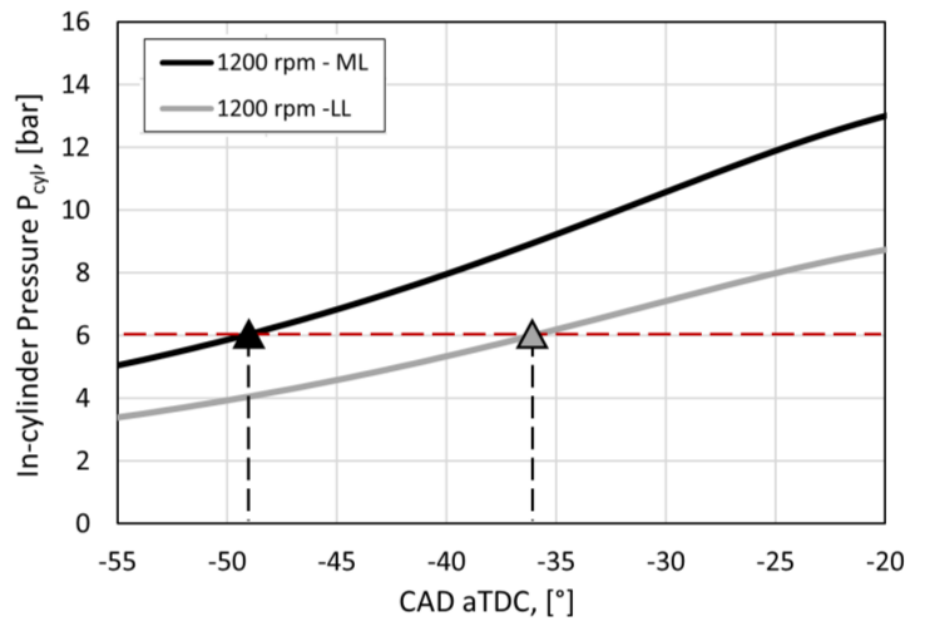

Fig.5. In-cylinder pressure evolution for ML case (black curve) and LL one (grey curve) at $1200 \mathrm{rpm}$. The corresponding ignition timings are respectively indicated through black triangle and grey one.

Other preliminary tests were deemed necessary in order to determine a $\mathrm{V}_{\mathrm{d}}$ common to all operating conditions which allows to avoid the streamer to arc transition [16]. The general aim is to maintain same discharge characteristics by fixing $\mathrm{V}_{\mathrm{d}}, \mathrm{t}_{\mathrm{on}}$ and $\mathrm{P}_{\text {cyl }}$ @ IT in order to investigate the streamer evolution under varying conditions. With safety margin to avoid desultory arcs, $\mathrm{V}_{\mathrm{d}}=24 \mathrm{~V}$ was chosen to execute the experimental campaign.

Fig.6 shows, for each CSI tip, the mean qualitative trend of the streamer penetration $\mathrm{P}$ during the igniter activation time, at $500 \mathrm{rpm}$ at LL. As it can be noticed, the $4^{\text {th }}$ quadrant is characterized by the longest penetration while the $3^{\text {rd }}$ presents the shortest one. It can be noted that the $1^{\text {st }}$ and $4^{\text {th }}$ tips, which featured the highest penetration on average, were the closest to the engine intake valves, while the $2^{\text {nd }}$ and $3^{\text {rd }}$ tips were the closest to the exhaust side. It is worth mentioning that the igniter orientation could affect such variability. In general, this kind of observation can be extended to the other engine speeds and loads analysed (Table2). 


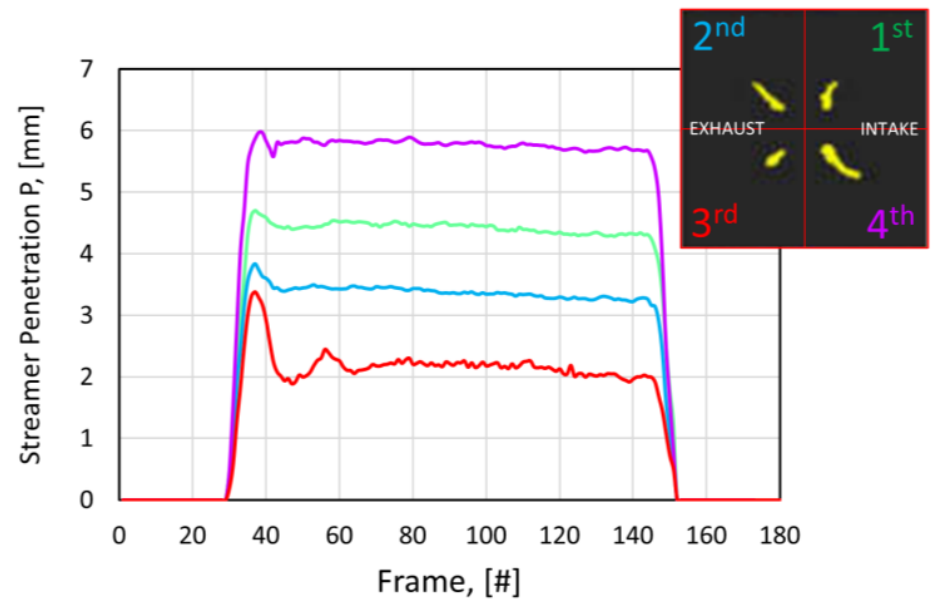

Fig.6. Mean streamer penetration evolution in the four quadrants at $500 \mathrm{rpm}$ and low load.

Since higher penetration results in a higher radical insemination and greater released thermal energy [18], it is possible to expect higher probability to ignite a mixture from $1^{\text {st }}$ and $4^{\text {th }}$ tips.

Fig.7 (a) shows the streamer evolution on the 1st quadrant, at each tested condition, focusing on the activation time (limited by black dotted lines, excluding the rising and falling transients) to highlight the penetration variation. In Fig.7 are reported the angular coefficients of the linear trend through which is possible to approximate the curve of Fig.7 (b) in the discharge interval. For sake of clarity, only the linear trend of the $1200 \mathrm{rpm}-\mathrm{ML}$ case is presented in Fig.7 (a). For both load cases, the streamer penetration progressively decreases as engine speed increases due to the higher-pressure levels in which the igniter is forced to work (see Table4). Moreover, at the same engine speed, the greater the load the higher the angular coefficient of the linear fit approximating the trends of Fig.7 (a). This behavior is probably related to the higher pressure characterizing the medium load due to the higher amount of air trapped inside the chamber.

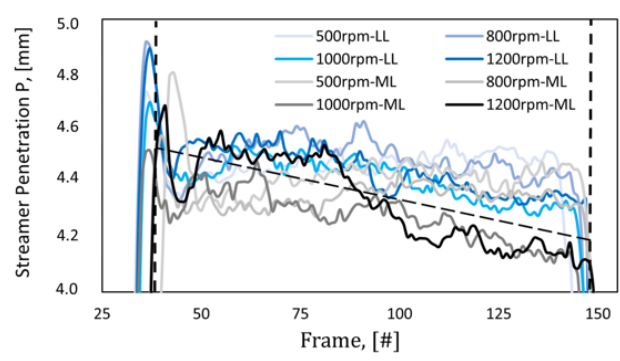

(a)

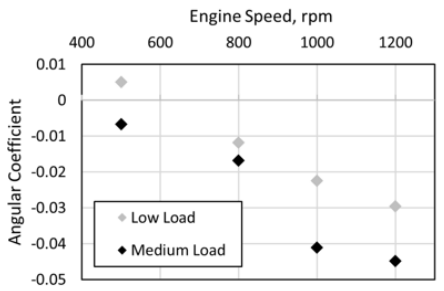

(b)

Fig.7. (a) Streamer penetration at the different cases analysed. For sake of clarity only the linear fit approximating the $1200 \mathrm{rpm}-\mathrm{ML}$ trend is presented. (b) Angular coefficients of the linear trend approximating the cases reported in (a) plotted against the engine speed (black markers for the Medium Load and grey for the Low Load).

Generally speaking, the previous observation can be extended to the other quadrants analysed. For what concerns the maximum penetration $\overline{P_{\max }}$ [18] there is no evidences of a direct relationship connecting $\overline{P_{\max }}$ to the engine speed or to the load. Moreover, the highest 
penetrations were found at the start of the discharge, i.e. at the lowest in-cylinder pressures. It is worth highlighting that this method did not take into account the angular position of the maximum penetration. Despite that, the effect of orientation on radical insemination and thermal energy release can be considered of second order with respect to streamer penetration length. Indeed, the latter results to be the key parameter affecting radical production and deposited thermal energy [21], and as a consequence, an effective combustion onset [22].

\subsection{Fired Condition}

A comparison between a motored and a lean operating condition is below proposed to analyse the mixture (air + gasoline) influence on the streamer evolution before ignition.

The low load motored-case at $1000 \mathrm{rpm}$ (Table 2) is compared to a lean-case [12] operating at the same speed with an air/fuel ratio equal to $1.6(\lambda=1.6)$ (Table5). It was found [12] that the operating point $\lambda=1.6$ represents the leanest stable condition characterizing the optical access engine of this work when operating with corona streamer-type igniter at $1000 \mathrm{rpm}$.

Table5. Technical characteristics of the compared motored and lean cases at $1000 \mathrm{rpm}$.

\begin{tabular}{|c|c|c|c|c|}
\hline Case & $\begin{array}{c}\text { Engine Speed, } \\
{[\mathbf{r p m}]}\end{array}$ & $\begin{array}{c}\mathbf{I T}, \\
\text { [CAD aTDC] }\end{array}$ & $\begin{array}{c}\mathbf{V}_{\mathrm{d}}, \\
\text { [CAD] }\end{array}$ & $\mathbf{P}_{\text {cyl }}$ (from-to), [bar] \\
\hline \multirow{2}{*}{ Lean $(\boldsymbol{\lambda}=1.6)$} & 1000 & -38 & 24 & $5.97-7.66$ \\
\cline { 3 - 5 } & & -49 & 19 & $4.41-5.80$ \\
\hline
\end{tabular}

In the lean-case, it was necessary to advance the ignition timing to guarantee a stable combustion. With respect to the motored-case, the lower $\mathrm{P}_{\text {cyl }}$ at the ignition timing requested lower $\mathrm{V}_{\mathrm{d}}$ to prevent streamer-to-arc transition. The differences in terms of streamer penetration P reported in Fig.8 between the motored (red curve) and lean case (black curve) are due to different $\mathrm{V}_{\mathrm{d}}$ and $\mathrm{P}_{\text {cyl }}$ conditions $[12,15]$.

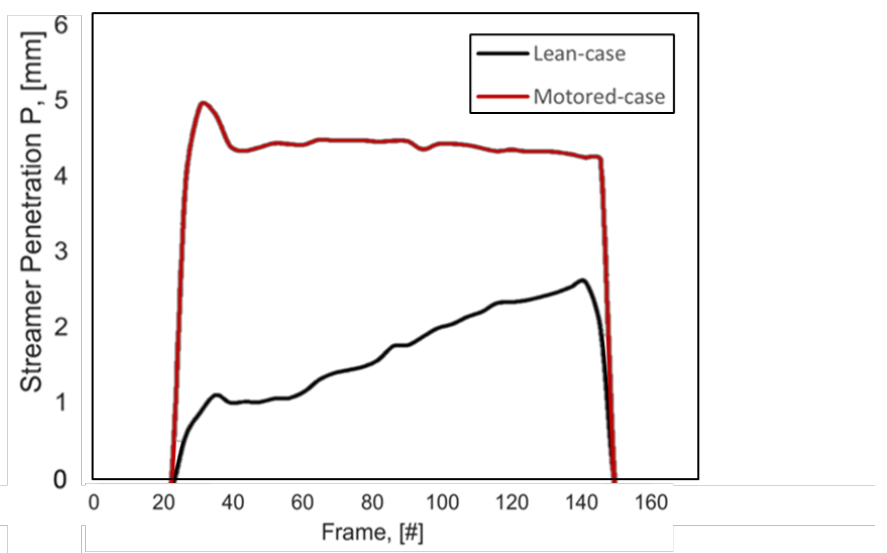

Fig.8. Streamer penetration for the motored-case (red curve) and lean one (black curve). For clarity, only the P of the $1^{\text {st }}$ quadrant is reported. Despite the differences in terms of streamer length found in the 4 quadrants (see Paragraph 5.1), the observations about the differences in the streamer evolution reported in this figure for the $1^{\text {st }}$ quadrant can be extended to the other tips.

Nevertheless, it could be interesting to focus the attention on the streamer evolution. At first, in the lean-case, the presence of gasoline emphasizes the local ionization of the 
mixture more than in the motored-case, thus damping the recorded brightness of the streamers [23]. In addition to that, in the motored-case the penetration progressively decreases as engine speed increases due to the higher-pressure levels, while in presence of gasoline (lean-operation) streamers penetration rises along the activation time. This effect could be linked to the chemical reactions that gradually reduce the hydrocarbons quenching effect [24], consequently promoting the streamer propagation.

Among all the streamer features, the penetration $\mathrm{P}$ parameter was chosen to investigate the behaviour of the CSI discharge inside the combustion chamber of an optical access engine. There is evidence that links the streamer penetration to the thermal energy released inside the combustion chamber [18,25] and to the production of radicals and excited species [26]. Therefore, since involving high amount of mixture during the discharge process is crucial to ensure robust combustions, the knowledge of streamers penetration gives the opportunity to evaluate the igniter capability to operate under critical operating conditions, as required by the increasingly stringent regulations on pollutant emissions.

At motored condition, the obtained results show that the maximum penetration does not seem be influenced by engine speed or load, which makes the igniter extremely suitable for different operating conditions. The influence of pressure, charge motion and temperature result to be more evident. As a matter of fact, higher penetrations were recorded @ the IT (lowest pressure) and in the coldest area of the chamber (tips $1^{\text {st }}$ and $4^{\text {th }}$ ).

The fired tests show that the mixture quality influence the streamer penetration much more than the other features. However, long activation times allow to progressively increase the streamers length, and with it, to enhance both volumetric and thermal effects. It is worth highlighting that, to make the most of these two effects, each engine operating point must be preliminarily optimized in terms of corona setting $\left(\mathrm{V}_{\mathrm{d},} \mathrm{T}_{\mathrm{on}}\right)$ and pressure @ the IT to avoid the streamer-to-arc transition phenomenon.

\section{Conclusions}

This work reports an analysis of the discharge natural luminosity of a RF corona igniter characterized by four tips electrodes in an optically accessible engine via high-speed camera detection. In the first part of the work, an investigation of the streamers evolution and penetration during the igniter activation time was performed in motored condition at four different engine speeds and two different loads, by setting the ignition timing at the same in-cylinder pressure value. The main results are listed below:

- For both load cases, the streamer penetration progressively decreases as engine speed increases due to the higher-pressure levels at which the igniter is forced to work.

- At the same engine speed, the higher the load the more pronounced the decreasing of the streamer penetration along the discharge, probably due to the higher pressure characterizing the medium load.

- In all cases analyzed, the tips featured with the highest penetration were the closest to the engine intake valves. The igniter orientation, together with the charge motion characterizing the optical engine and the higher local temperature close to the exhaust side, could affect such variability.

- There is no evidence of a direct relationship linking the maximum streamer penetration to the engine speed and/or to the load.

In the second part of the work, the low load motored case at $1000 \mathrm{rpm}$ was compared to a lean-case operating at the same speed with an air/fuel ratio equal to 1.6 to analyze the mixture influence on the streamer evolution before the ignition.

- Despite the differences in terms of streamer penetration length between the two analyzed cases, in the motored one the streamer penetration progressively decreases as 
engine speed increases, due to the higher-pressure levels, while in the lean-case the penetration rises along with the activation time. This effect could be linked to the chemical reactions that gradually reduce the hydrocarbons quenching effect, consequently promoting the streamer propagation.

\section{Glossary and nomenclature}

$\begin{array}{ll}\text { ACIS } & \text { advanced corona ignition system } \\ \text { ICE } & \text { internal combustion engine } \\ \text { IT } & \text { ignition timing } \\ \text { LTP } & \text { low temperature plasma } \\ \lambda & \text { air-fuel ratio } \\ \mathrm{t}_{\text {on }} & \text { corona discharge duration } \\ \mathrm{V}_{\text {ch }} & \text { vessel inner volume } \\ \mathrm{V}_{\mathrm{d}} & \text { corona driving voltage } \\ \mathrm{V}_{\mathrm{e}} & \text { peak electrode voltage } \\ \mathrm{P}_{\mathrm{P}} & \text { streamer penetration } \\ \mathrm{P}_{\text {cyl }} & \text { in-cylinder pressure }\end{array}$

\section{Funding}

This research activity was partially granted by the Basic Research Funds supplied by the Department of Engineering of the University of Perugia.

\section{References}

1. D. Takahashi et al. "Combustion Development to Realize High Thermal Efficiency Engines", SAE Int. J. Engines 9, 2016-01-0693 (2016).

2. T. Johnson and A. Joshi. "Review of Vehicle Engine Efficiency and Emissions", SAE Int. J. Engines 11, 2018-01-0329 (2018).

3. S. Zhu et al., "A review of water injection applied on the internal combustion engine," Energy Convers. Manag,vol. 184, no. January, pp. 139-158, 2019.

4. C. Gong, Z. Zhang, J. Sun, Y. Chen, and F. Liu, "Computational study of nozzle spray-line distribution effects on stratified mixture formation, combustion and emissions of a high compression ratio DISI methanol engine under lean-burn condition", Energy, vol. 205, p. 118080, 2020.

5. V. Cruccolini et al., "Lean combustion analysis using a corona discharge igniter in an optical engine fueled with methane and a hydrogen-methane blend," Fuel, vol. 259, no. September 2019, p. 116290, 2020.

6. Abidin, Z. and Chadwell, C., "Parametric Study and Secondary Circuit Model Calibration Using Spark Calorimeter Testing," SAE Technical Paper.

7. D. Jung and N. Iida, "An investigation of multiple spark discharge using multi-coil ignition system for improving thermal efficiency of lean SI engine operation", Appl. Energy, vol. 212, no. December 2017, pp. 322-332, 2018. https://doi.org/10.1016/j.apenergy.2017.12.032

8. Jung D, Sasaki K, Iida N. "Effects of increased spark discharge energy and enhanced in-cylinder turbulence level on lean limits and cycle-to-cycle variations of combustion for SI engine operation.", Appl Energy 2017;205(August):1467-77. https:// doi.org/10.1016/j.apenergy.2017.08.043.

9. Idicheria, C.A., Yun, H., and Najt, P.M., "An Advanced Ignition System for High Efficiency Engines," Ignition Systems for Gasoline Engines : 4th International Conference, December 6 - 7, 2018, Berlin, Germany, 40-54, 2018.

10. Shiraishi, T. and Urushihara, T., "Fundamental Analysis of Combustion Initiation Characteristics of Low Temperature Plasma Ignition for Internal Combustion Gasoline Engine," SAE Technical Paper, 399-408, 2011.

11. Starikovskaia, S. M., "Plasma assisted ignition and combustion." Journal of Physics D: Applied Physics: 39:R265-R299, 2006. doi:10.1088/0022-3727/39/16/R0. 
12. Cruccolini, V., Discepoli, G., Ricci, F., Petrucci, L. et al., "Comparative Analysis between a Barrier Discharge Igniter and a Streamer-Type Radio-Frequency Corona Igniter in an Optically Accessible Engine in Lean Operating Conditions," SAE Technical Paper 2020-01-0276, 2020.

13. F. Ricci, J. Zembi, M. Battistoni, C. Grimaldi, and G. Discepoli, "Experimental and Numerical Investigations of the Early Flame Development Produced by a Corona Igniter," SAE Technical Paper 2019.

14. V. Cruccolini et al., "Multidimensional modeling of non-equilibrium plasma generated by a radiofrequency corona discharge," Plasma Sources Sci. Technol., vol. 29, no. 11, p. 115013, Nov. 2020.

15. Ricci, F., Petrucci, L., Cruccolini, V., Discepoli, G., Grimaldi, C.N., and Papi, S., "Investigation of the Lean Stable Limit of a Barrier Discharge Igniter and of a Streamer-Type Corona Igniter at Different Engine Loads in a Single-Cylinder Research Engine," Proceedings 58(1):11, 2020.

16. G. Discepoli, V. Cruccolini, F. Ricci, A. Di Giuseppe, S. Papi, and C. N. Grimaldi, "Experimental characterisation of the thermal energy released by a Radio- Frequency Corona Igniter in nitrogen and air", Appl. Energy, vol. 263, no. February, p. 114617, 2020.

17. Discepoli, G.; Cruccolini, V.; Dal Re, M.; Zembi, J.; Battistoni, M.; Mariani, F.; Grimaldi, C.N. Experimental Assessment of Spark and Corona Igniters Energy Release. Energy Procedia 2018, 148, 1262-1269.

18. V. Cruccoliniet al., 'An Optical Method to Characterize Streamer Variability and Streamer-to-Flame Transition for Radio-Frequency Corona Discharges', AppSci 2020.

19. Jung D, Sasaki K, Iida N. "Effects of increased spark discharge energy and enhanced in-cylinder turbulence level on lean limits and cycle-to cycle variations of combustion for SI engine operation.", Appl Energy 2017.

20. A. Cimarello,et al., "Analysis of RF Corona Ignition in Lean Operating Conditions Using an Optical Access Engine”, SAE Technical Paper 2017.

21. Wolk, B.M.; Ekoto, I. Calorimetry and Imaging of Plasma Produced by a Pulsed Nanosecond Discharge Igniter in EGR Gases at Engine-Relevant Densities. SAE Int. J. Engines 2017.

22. Ju, Y.; Sun, W. Plasma assisted combustion: Dynamics and chemistry.

23. A. You, M. A. Y. Be, and I. In, "The variation of ionization with air / fuel ratio for a spark-ignition engine," vol. 505, no. August, 2008.

24. C. S. Parmenter and J. D. Rau, "Fluorescence quenching in aromatic hydrocarbons by oxygen," J. Chem. Phys., vol. 51, no. 5, pp. 2242-2246, 1969.

25. F. Ricci et al., "Energy characterization of an innovative non-equilibrium plasma ignition system based on the dielectric barrier discharge via pressure-rise calorimetry," Energy Convers. Manag., vol. 244, no. April, p. 114458, 2021.

26. Scarcelli, R.; Wallner, T.; Som, S.; Biswas, S.; Ekoto, I.; Breden, D.; Karpatne, A.; Raja, L. Modeling NonEquilibrium Discharge and Validating Transient Plasma Characteristics at Above-Atmospheric Pressure. Plasma Source Sci. Technol. 2018, 27, 124006, doi:10.1088/1361-6595/aaf539. 